\title{
Desenvolvimento e validação de equação para determinação do coeficiente de dispersão longitudinal em rios de médio porte
}

\section{Development and validation of an equation to estimate longitudinal dispersion coefficient in medium-sized rivers}

\begin{abstract}
Celso Bandeira de Melo Ribeiro
Engenheiro Civil. Mestre em Engenharia Civil pelo Instituto Alberto Luiz Coimbra de Pós-graduação e Pesquisa de Engenharia da Universidade Federal do Rio de Janeiro (COPPE/UFRJ). Doutor em Engenharia Agrícola pela Universidade Federal de Viçosa (UFV). Professor Adjunto I do Departamento de Engenharia Sanitária e Ambiental da Faculdade de Engenharia da Universidade Federal de Juiz de Fora (UFJF)
\end{abstract}

\section{Demetrius David da Silva}

Engenheiro Agrônomo. Mestre e Doutor em Engenharia Agrícola pela UFV. Professor Associado III da UFV. Bolsista de Produtividade em Pesquisa do CNPq - Nível $1 \mathrm{~A}$

\section{José Homero Pinheiro Soares}

Engenheiro Civil. Mestre e Doutor em Engenharia Civil pela COPPE/UFRJ. Professor Adjunto III da UFJF

\section{Hugo Alexandre Soares Guedes}

Engenheiro Civil. Doutorando em Engenharia Agrícola pela UFV

\section{Resumo}

No presente trabalho, desenvolveu-se equação para predição do coeficiente de dispersão longitudinal ( $\left.D_{\llcorner}\right)$em rios de médio porte, com vazões entre 16,20 e $98 \mathrm{~m}^{3} \mathrm{~s}^{-1}$, a partir de ensaios experimentais utilizando traçadores fluorescentes. A equação foi deduzida utilizando-se análise dimensional e ajustada aos dados de campo pela técnica de regressão linear múltipla. Em seguida, realizou-se a validação da equação testando-a em outra base de dados, diferente daquela para a qual foi desenvolvida, com vazões entre 19,57 a 48,54 $\mathrm{m}^{3} \mathrm{~s}^{-1}$. Adicionalmente, foram comparados os desempenhos de outras quatro equações empíricas de predição do $D_{L}$, utilizando-se três métodos comparativos. A equação desenvolvida apresentou ajuste estatístico adequado e bom resultado, com melhor desempenho geral quando comparada a outras equações propostas na literatura.

Palavras-chave: dispersão longitudinal; poluição hídrica; qualidade da água

\begin{abstract}
In this paper, an equation has been developed to estimate the longitudinal dispersion coefficient $\left(D_{L}\right)$ in medium-sized rivers, with flow between 16.20 and $98 \mathrm{~m}^{3} \mathrm{~s}^{-1}$, from experimental tests using fluorescent tracers. The equation was deduced by dimensional analysis and adjusted to the field data by the multiple linear regression technique. Thereafter, the validation of the equation was performed by testing it in another database, which is different from the one it had been developed for, with flow between 19.57 and $48.54 \mathrm{~m}^{3} \mathrm{~s}^{-1}$. Additionally, the performance of four other empirical equations was compared for $\mathrm{D}_{\mathrm{L}}$ prediction using three comparative methods. The developed equation showed appropriate statistical adjustment and good result, with better overall performance when compared with other equations proposed in literature.
\end{abstract}

Keywords: longitudinal dispersion; water pollution; water quality. 


\section{Introdução}

A quantificação de parâmetros de transporte e dispersão de poluentes solúveis nos cursos d'água naturais possui fundamental importância no gerenciamento dos recursos hídricos e em estudos que utilizam modelos de qualidade de água, uma vez que levam em consideração mecanismos de transporte advectivo e difusivo de poluentes na massa hídrica.

Uma forma reconhecidamente adequada para se determinar o coeficiente de dispersão longitudinal $\left(\mathrm{D}_{\mathrm{L}}\right)$ de efluentes em rios é a partir de experimentos com traçadores, conforme apresentam os estudos desenvolvidos por Ribeiro et al (2008) e Pereira (1999); entretanto, sua utilização requer grande esforço na realização dos ensaios in situ.

Nesse contexto, as equações empíricas apresentam grande utilidade na predição do $D_{L}$, uma vez que facilitam sua obtenção a partir de poucos parâmetros relacionados às características de escoamento do curso d'água.

Nessas equações, de uma forma geral, o $D_{L}$ é estimado em função das características do rio, expressas normalmente por largura (B), profundidade $(\mathrm{H})$, velocidade média do escoamento (U), velocidade de cisalhamento $\left(u^{*}\right)$ e massa específica do fluido ( $\rho$ ) (DEVENS; BARBOSA JUNIOR; SILVA, 2006).

Diversas fórmulas empíricas de predição do $\mathrm{D}_{\mathrm{L}}$ são citadas na literatura, conforme apresentado por Ribeiro et al (2007), Devens, Barbosa Junior e Silva (2006), Tayfur e Singh (2005), Rieckermann et al (2004), Seo e Cheong (1998) e Bowie et al (1985). Contudo, essas equações foram deduzidas para condições específicas de escoamento, limitando sua aplicabilidade a condições hidráulicas semelhantes.

Diante do exposto, este trabalho teve como objetivo desenvolver uma equação para predição do $\mathrm{D}_{\mathrm{L}}$, a partir de resultados experimentais, utilizando-se traçadores fluorescentes (Uranina e Amidorodamina G Extra) em rios de médio porte, com vazão entre 16,20 e $98 \mathrm{~m}^{3} \mathrm{~s}^{-1}$, localizados na região Sudeste do Brasil, visando facilitar a obtenção do $D_{L}$ para condições semelhantes. Procurou-se validar a equação testando-a em outra base de dados, diferente daquela para a qual foi desenvolvida.

Vale ressaltar que o desenvolvimento e a validação de uma equação para rios de médio porte contribuem expressivamente para trabalhos de modelagem da qualidade da água, em regimes hidrológicos semelhantes, devido à simplicidade de obtenção do $\mathrm{D}_{\mathrm{L}}$, quando comparados aos métodos de campo, que exigem equipamentos e técnicos especializados, além de demandarem recursos expressivos para realização do ensaio in situ e análise de amostras em laboratório.

\section{Metodologia}

A área de estudo na qual foram realizados os experimentos de campo para a determinação do $\mathrm{D}_{\mathrm{L}}$ compreende as bacias hidrográficas dos rios Pomba e Paraibuna, localizadas na região da Zona da Mata Mineira (Figura 1). Ressalta-se que essas bacias são adjacentes e pertencem à bacia do rio Paraíba do Sul, na sua parte mineira, em quase sua totalidade.

O método adotado para determinação do $\mathrm{D}_{\mathrm{L}}$ baseou-se na injeção instantânea do corante e posterior monitoramento euleriano das variações temporais das concentrações do traçador em cada seção de monitoramento, possibilitando determinar as curvas de passagem nessas seções (RIBEIRO et al, 2007). Os cálculos para a determinação do $\mathrm{D}_{\mathrm{L}}$ foram realizados com o ajuste de um modelo Fickiano aos valores obtidos nas curvas de passagem do traçador. Esse modelo, apresentado na Equação 1, foi desenvolvido com base na equação de transporte de massa por mecanismos de advecção e dispersão na direção longitudinal, considerandose regime de escoamento permanente e uniforme e constituinte conservativo.

$C(x, t)=\frac{M}{A \sqrt{4 \pi \cdot D_{L} \cdot t}} \exp \frac{-(x-U t)^{2}}{4 D_{L} t}$ Equação 1

Onde:

C: concentração do constituinte ou traçador $\left(\mathrm{M} \mathrm{L}^{-3}\right)$;

M: massa de constituinte injetada (M);

A: área da seção transversal ( $\left.\mathrm{L}^{2}\right)$;

$\mathrm{D}_{\mathrm{L}}$ : coeficiente de dispersão longitudinal $\left(\mathrm{L}^{2} \mathrm{~T}^{-1}\right)$;

$\mathrm{U}$ : velocidade do escoamento ( $\mathrm{L} \mathrm{T}^{-1}$ );

x e t: variáveis espacial (L) e temporal (T), respectivamente.

As características hidrológicas dos cursos d'água, para os locais de monitoramento utilizados na dedução da equação, foram obtidas a partir das curvas de descarga das estações fluviométricas 58735000 (Astolfo Dutra), 58770000 (Cataguases), ambas no rio Pomba, e 58480500 (Juiz de Fora Jusante), no rio Paraibuna. Os valores de dispersão longitudinal do escoamento foram obtidos a partir do ajuste do modelo Fickiano às curvas de passagem dos traçadores Uranina e Amidorodamina G Extra pelas seções de monitoramento. Já as informações de declividade foram obtidas a partir de técnicas de geoprocessamento, com base no modelo digital de elevação do terreno, oriundo de dados SRTM (Shuttle Radar Topography Mission), disponibilizado pela NASA.

A Tabela 1 apresenta as características hidrogeométricas e os valores de $\mathrm{D}_{\mathrm{L}}$ obtidos em oito experimentos de campo realizados nos rios Pomba e Paraibuna. Esses valores foram utilizados para o desenvolvimento da equação de predição do $\mathrm{D}_{\mathrm{L}}$.

\section{Desenvolvimento da equação}

No desenvolvimento da equação para determinação do $\mathrm{D}_{\mathrm{L}}$, foram aplicadas as metodologias utilizadas por Devens, Barbosa Junior e Silva (2006) e Seo e Cheong (1998). De acordo com os estudos 


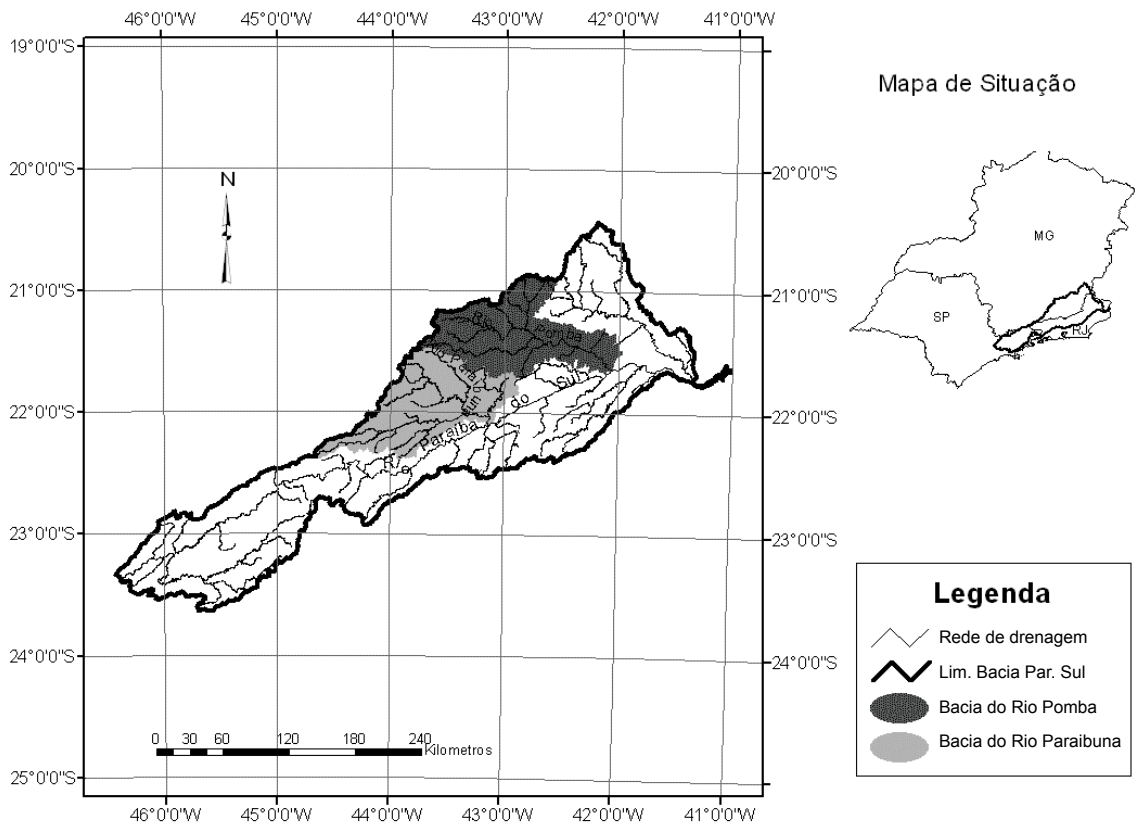

Lim. Bacia Par. Sul: Limite da Bacia do Rio Paraíba do Sul.

Figura 1 - Localização das bacias hidrográficas dos rios Pomba e Paraibuna

Tabela 1 - Valores do coeficiente de dispersão longitudinal $\left(D_{1}\right)$ e características hidrogeométricas obtidas em experimentos realizados nos rios Pomba e Paraibuna, utilizando-se injeção instantânea de traçadores fluorescentes Uranina (URA) e Amidorodamina G Extra (AMI)

\begin{tabular}{lccccccccccccc} 
Rio/Estação Fluv. & Data & Injeção & $\begin{array}{c}\mathrm{DL} \\
\left(\mathrm{m}^{2} / \mathrm{s}\right)\end{array}$ & $\begin{array}{c}\mathrm{Q} \\
\left(\mathrm{m}^{3} / \mathrm{s}\right)\end{array}$ & $\begin{array}{c}\mathrm{B} \\
(\mathrm{m})\end{array}$ & $\begin{array}{c}\mathrm{U} \\
(\mathrm{m} / \mathrm{s})\end{array}$ & $\begin{array}{c}\mathrm{H} \\
(\mathrm{m})\end{array}$ & $\begin{array}{c}\mathrm{S} \\
(\mathrm{m} / \mathrm{m})\end{array}$ & $\begin{array}{c}\mathbf{u}^{*} \\
(\mathrm{~m} / \mathrm{s})\end{array}$ & $\begin{array}{c}\mathrm{DL} / \\
\left(\mathrm{u}^{*} \mathrm{H}\right)\end{array}$ & $\begin{array}{c}\mathrm{B} / \mathrm{H} \\
\mathbf{u}^{*} / \mathrm{U}\end{array}$ & $\mathrm{Re}^{*}$ \\
\hline Paraibuna/JF Jus. & $10 / 21 / 1995$ & $\mathrm{URA} / 20,0$ & 35,00 & 42,60 & 26,00 & 0,92 & 1,79 & 0,001 & 0,09 & 214,34 & 14,53 & 0,10 & 163295 \\
\hline Paraibuna/JF Jus. & $1 / 23 / 1996$ & $\mathrm{AMI} / 20,0$ & 10,00 & 19,60 & 25,50 & 0,66 & 1,17 & 0,001 & 0,08 & 113,50 & 21,79 & 0,11 & 88103 \\
\hline Paraibuna/JF Jus. & $5 / 21 / 1996$ & $\mathrm{AMI} / 20,0$ & 8,50 & 16,20 & 21,00 & 0,57 & 1,36 & 0,001 & 0,08 & 78,34 & 15,44 & 0,14 & 108501 \\
\hline Paraibuna/JF Jus. & $10 / 7 / 2005$ & $\mathrm{URA} / 20,0$ & 12,00 & 23,20 & 23,00 & 0,77 & 1,31 & 0,001 & 0,08 & 116,06 & 17,56 & 0,10 & 103398 \\
\hline Paraibuna/JF Jus. & $12 / 1 / 2005$ & $\mathrm{AMI} / 20,0$ & 15,00 & 33,03 & 28,00 & 0,83 & 1,43 & 0,001 & 0,08 & 126,46 & 19,58 & 0,10 & 118613 \\
\hline Pomba/Ast. Dutra & $5 / 28 / 2006$ & $\mathrm{AMI} / 6,0$ & 19,00 & 38,80 & 44,00 & 0,65 & 1,34 & 0,002 & 0,14 & 98,52 & 32,84 & 0,22 & 192846 \\
\hline Pomba/Cataguases & $5 / 28 / 2006$ & $\mathrm{AMI} / 36,0$ & 33,00 & 98,00 & 81,00 & 0,50 & 2,42 & 0,002 & 0,20 & 68,47 & 33,47 & 0,40 & 481982 \\
Pomba/Ast.Dutra & $4 / 14 / 2007$ & $\mathrm{AMI} / 6,0$ & 19,00 & 36,00 & 40,00 & 0,65 & 1,37 & 0,002 & 0,15 & 95,21 & 29,20 & 0,23 & 199563
\end{tabular}

1 Distância em quilômetros considerada a partir do ponto de injeção de traçador.

Q: vazão; B: largura; U: velocidade média do escoamento; H: profundidade; S: declividade do curso d'água; u*: velocidade de cisalhamento; Re*: número de Reynolds; JF Jus: Juiz de Fora Jusante; Ast. Dutra: Astolfo Dutra.

desenvolvidos por esses autores, os fatores que mais influenciam a dispersão $\left(D_{L}\right)$ de poluentes em escoamentos naturais são classificados em três grupos: a) propriedades dos fluidos representadas pela sua massa específica e viscosidade; b) características hidráulicas de escoamento, representadas pela velocidade média na seção, velocidade de cisalhamento, largura e profundidade do canal; e c) configuração geométrica do curso d'água representada pela sinuosidade e forma do leito do canal.

Sendo assim, foram utilizados seis parâmetros $\left(\rho, v, U, u^{*}\right.$, $H, B$ ) para o desenvolvimento da equação de predição do $D_{L}$. Tomando-se o valor de $\mathrm{D}_{\mathrm{L}}$ como variável dependente, pode-se expressar a relação entre as variáveis representativas conforme mostra a Equação 2.

$D_{L}=f_{1}\left(\rho, v, U, u^{*}, H, B\right)$
Onde:

$\mathrm{D}_{\mathrm{L}}$ : coeficiente de dispersão longitudinal $\left(\mathrm{L}^{2} \mathrm{~T}^{-1}\right)$;

$\rho$ : densidade absoluta ou massa específica do fluido $\left(\mathrm{ML}^{-3}\right)$;

$v$ : viscosidade cinemática do fluido $\left(\mathrm{L}^{2} \mathrm{~T}^{-1}\right)$;

U: velocidade média na seção $\left(\mathrm{LT}^{-1}\right)$;

$\mathrm{u}^{*}$ : velocidade de cisalhamento $\left(\mathrm{LT}^{-1}\right)$;

B e H: largura e profundidade média do canal, respectivamente (L).

Aplicando-se o teorema de Vaschy-Buckingham ou teorema dos $\pi$, foram escolhidas as dimensões $\mathrm{M}$, L e T como fundamentais na especificação dos sete parâmetros envolvidos: $\left[\mathrm{D}_{\mathrm{L}}\right]=\mathrm{L}^{2} \mathrm{~T}^{-1} ;[\rho]=$ $\mathrm{ML}^{-3} ;[v]=\mathrm{L}^{2} \mathrm{~T}^{-1} ;[\mathrm{U}]=\mathrm{LT}^{-1} ;\left[\mathrm{u}^{*}\right]=\mathrm{LT}^{-1} ;[\mathrm{H}]=\mathrm{L} ;[\mathrm{B}]=\mathrm{L}$. Seguindose a metodologia adotada por Devens, Barbosa Junior e Silva (2006) foram escolhidos os parâmetros $\rho, u^{*}$ e H como grandezas básicas ou parâmetros repetitivos. Com sete parâmetros dimensionais e 
três repetitivos, restaram quatro grupos adimensionais, conforme a Equação 3.

$\pi_{1}=\mathrm{f}_{2}\left(\pi_{2}, \pi_{3}, \pi_{4}\right)$

Equação 3

Escrevendo-se os parâmetros $\pi$ na forma de expoentes a determinar, resolvendo o sistema de equações e substituindo os resultados na expressão dos $\pi$ (Equação 3), chega-se aos grupos adimensionais apresentados na Equação 4

$\frac{\mathrm{D}_{\mathrm{L}}}{\mathrm{u}^{*} \mathrm{H}}=\mathrm{f}_{3}\left(\frac{\mathrm{B}}{\mathrm{H}}, \frac{\mathrm{u}^{*}}{\mathrm{U}}, \mathrm{Re}^{*}\right)$

Equação 4

Onde $\operatorname{Re}^{*}=\frac{\mathrm{u}^{*} \cdot \mathrm{H}}{\mathrm{n}}$ é o número de Reynolds considerando-se a velocidade de cisalhamento $\left(u^{*}=\sqrt{g \cdot R_{H} \cdot S}\right)$.

Cabe ressaltar que o Re* diferencia-se do número de Reynolds tradicional (Re), usado em condutos livres e forçados, que utiliza a velocidade média das seções de monitoramento.

Após definir os grupos adimensionais entre os principais parâmetros que influenciam na determinação do $\mathrm{D}_{\mathrm{L}}$, conforme análise dimensional (Equação 4), foi adotado o modelo potencial (Equação 5) para descrever a relação de dependência entre as variáveis.

$\frac{\mathrm{D}_{\mathrm{L}}}{\mathrm{u}^{*} \mathrm{H}}=\mathrm{n}_{0}\left(\frac{\mathrm{B}}{\mathrm{H}}\right)^{\mathrm{n}_{1}}\left(\frac{\mathrm{u}^{*}}{\mathrm{U}}\right)^{\mathrm{n}_{2}}\left(\operatorname{Re}^{*}\right)^{\mathrm{n}_{3}}$

Equação 5

A estimativa dos parâmetros $\beta\left(\beta_{0}, \beta_{1} \beta_{2}\right.$ e $\left.\beta_{3}\right)$ foi realizada por meio de ajuste de regressão linear múltipla aos dados experimentais das duas bacias (Pomba e Paraibuna), apresentados na Tabela 1. Para verificar a qualidade do modelo ajustado aos dados de campo, os resultados foram analisados estatisticamente com base no índice de determinação $\left(\mathrm{r}^{2}\right)$ e no teste de F, utilizando-se o Sistema para Análises Estatísticas (SAEG), versão 9.1, desenvolvido pela Universidade Federal de Viçosa, Minas Gerais.

\section{Validação e comparação do desempenho da equação desenvolvida}

Para validar a aplicabilidade da equação desenvolvida neste trabalho a outras condições de campo, diferentes daquelas utilizadas em sua dedução, esta foi testada com base em resultados experimentais obtidos por Bandeira (2004) no rio das Velhas, afluente do Rio São Francisco, região metropolitana de Belo Horizonte, Minas Gerais, e por Ribeiro et al (2008) nos rios Pomba e Paraibuna, afluentes do Rio Paraíba do Sul, região da Zona da Mata Mineira. Realizou-se também a comparação do desempenho entre quatro equações propostas na literatura com a equação desenvolvida no trabalho, utilizando-se como base os resultados dos coeficientes de dispersão longitudinal obtidos nesses experimentos. Na Tabela 2, são apresentadas as equações empíricas utilizadas para fins de comparação.

Foram utilizados na comparação a razão de discrepância $\left(R_{d}\right)$ a raiz do erro médio quadrático (REMQ) e o índice de desempenho (c), proposto por Camargo e Sentelhas (1997), adotando-se como valores observados ou medidos $\left(\mathrm{O}_{\mathrm{i}}\right)$ aqueles obtidos por Bandeira (2004) e Ribeiro et al (2008) por meio de técnicas de traçadores (Tabela 3), tendo sido utilizados o método da convolução e o ajuste do modelo Fickiano de Taylor, respectivamente, para determinação do $\mathrm{D}_{\mathrm{L}}$; e como valores estimados $\left(\mathrm{E}_{\mathrm{i}}\right)$, utilizaram-se aqueles obtidos por cada uma das fórmulas empíricas apresentadas na Tabela 2, bem como o valor da equação desenvolvida no presente trabalho.

A razão de discrepância $\left(R_{d}\right)$, definida por White et al (1973) (apud SEO; CHEONG, 1998), é dada pela Equação 6.

$\mathrm{R}_{\mathrm{d}}=\log \frac{\mathrm{E}_{\mathrm{i}}}{\mathrm{O}_{\mathrm{i}}}$

Equação 6

Onde: $\mathrm{E}_{\mathrm{i}}$ são os valores estimados de $\mathrm{D}_{\mathrm{L}}\left(\mathrm{L}^{2} \mathrm{~T}^{-1}\right)$ e $\mathrm{O}_{\mathrm{i}}$ são os valores observados de $\mathrm{D}_{\mathrm{L}}\left(\mathrm{L}^{2} \mathrm{~T}^{-1}\right)$ para o i-ésimo evento, respectivamente.

Tabela 2 - Fórmulas empíricas de predição do coeficiente de dispersão longitudinal $\left(D_{\llcorner}\right)$em rios e canais abertos

\begin{tabular}{|c|c|c|c|}
\hline Autor/ano & & Equação ${ }^{1}$ & $\begin{array}{l}\text { Faixa de variação de velocidade }(U) \text { e profundidade média de escoa- } \\
\text { mento }(H)\end{array}$ \\
\hline $\begin{array}{l}\text { Seo e Cheong } \\
(1998)\end{array}$ & Equação 6 & $\mathrm{D}_{\mathrm{L}}=5,915 \cdot\left(\mathrm{H} \cdot \mathrm{u}^{*}\right) \cdot\left(\frac{\mathrm{B}}{\mathrm{H}}\right)^{0,62} \cdot\left(\frac{\mathrm{U}}{\mathrm{u}^{*}}\right)^{1,428}$ & $\begin{array}{l}\text { A faixa de variação da velocidade média de escoamento e profundi- } \\
\text { dade utilizados na sua dedução são } 0,13 \text { a } 1,74 \mathrm{~m} / \mathrm{s} \text { e } 0,22 \text { a } 19,94 \mathrm{~m} \text {, } \\
\text { respectivamente. }\end{array}$ \\
\hline $\begin{array}{l}\text { Deng, Singh e } \\
\text { Bengtsson (2001) }\end{array}$ & Equação 7 & $\begin{array}{l}\mathrm{D}_{\mathrm{L}}=0,15 \cdot\left(\frac{\mathrm{H} \cdot \mathrm{u}^{*}}{8 \cdot \mathrm{n}_{\mathrm{t} 0}}\right) \cdot\left(\frac{\mathrm{B}}{\mathrm{H}}\right)^{5 / 3}\left(\frac{\mathrm{U}}{\mathrm{u}^{*}}\right)^{2} \\
\mathrm{n}_{\mathrm{t} 0}=0,145+\left(\frac{1}{3520}\right)\left(\frac{\mathrm{U}}{\mathrm{u}^{*}}\right)\left(\frac{\mathrm{B}}{\mathrm{H}}\right)^{1,38}\end{array}$ & $\begin{array}{l}\text { Utilizou a mesma base de dados da Equação } 6 \text { para sua dedução. } \\
\text { Assim, a faixa de variação da velocidade média de escoamento e } \\
\text { profundidade utilizados na sua dedução são } 0,13 \text { a 1,74 m/s e } 0,22 \text { a } \\
19,94 \text { m, respectivamente. }\end{array}$ \\
\hline $\begin{array}{l}\text { Kashefipour e } \\
\text { Falconer } \\
\text { (2002) }\end{array}$ & Equação 8 & $\mathrm{D}_{\mathrm{L}}=10,612 \cdot(\mathrm{H} \cdot \mathrm{U}) \cdot\left(\frac{\mathrm{U}}{\mathrm{u}^{*}}\right)$ & $\begin{array}{l}\text { A faixa de variação da velocidade média de escoamento e profundi- } \\
\text { dade utilizados na sua dedução são } 0,14 \text { a 1,55 m/s e } 0,26 \text { a 4,75 m, } \\
\text { respectivamente. }\end{array}$ \\
\hline $\begin{array}{l}\text { Devens, Barbosa } \\
\text { Junior e Silva (2006) }\end{array}$ & Equação 9 & $\mathrm{D}_{\mathrm{L}}=3,55 \times 10^{-4} \frac{\mathrm{U}^{-0,793} \cdot \mathrm{B}^{0,739}}{\mathrm{H}^{1,610} \cdot \mathrm{S}_{0}^{0,026}}$ & $\begin{array}{l}\text { Desenvolvida para pequenos cursos d'água naturais com vazões } \\
\text { entre } 0,00521 \text { a } 0,173 \mathrm{~m}^{3} / \mathrm{s} \text {. A faixa de variação da velocidade média } \\
\text { de escoamento e profundidade utilizados na sua dedução são } 0,08 \text { a } \\
0,34 \mathrm{~m} / \mathrm{s} \text { e } 0,02 \text { a } 0,10 \mathrm{~m} \text {, respectivamente. }\end{array}$ \\
\hline
\end{tabular}

${ }^{1} \mathrm{H}$ : profundidade média da seção $(\mathrm{L})$; $\mathrm{u}^{*}$ : velocidade de cisalhamento $\left(\mathrm{LT}^{-1}\right)$; $\mathrm{B}$ : largura da seção transversal do curso d'água $(\mathrm{L}) ; \boldsymbol{\varepsilon}_{t 0}$ : coeficiente de difusão transversal ( $\left.\mathrm{L}^{2} \mathrm{~T}^{-1}\right)$; $\mathrm{S}_{0}$ : declividade do leito $\left(L . L^{-1}\right)$. 
Tabela 3 - Valores de D e características hidrogeométricas obtidos por Bandeira (2004), a partir de experimentos com injeção contínua de traçador fluorescente Rodamina WT (R0), e por Ribeiro et al (2008), utilizando-se injeção instantânea de traçador fluorescente Amidorodamina G Extra (AMI)

\begin{tabular}{lcccccccccc}
$\begin{array}{c}\text { Observação } \\
\text { (i) }\end{array}$ & Rio / Seção & Data & $\begin{array}{c}\text { Traçador/ } \\
\text { Dist. }(\mathrm{km})\end{array}$ & $\begin{array}{c}\mathrm{DL} \\
\left(\mathrm{m}^{2} / \mathrm{s}\right)\end{array}$ & $\begin{array}{c}\mathbf{Q} \\
\left(\mathrm{m}^{3} / \mathrm{s}\right)\end{array}$ & $\begin{array}{c}\mathrm{B} \\
(\mathrm{m})\end{array}$ & $\begin{array}{c}\mathbf{U} \\
(\mathrm{m} / \mathrm{s})\end{array}$ & $\begin{array}{c}\mathbf{H} \\
(\mathrm{m})\end{array}$ & $\begin{array}{c}\mathrm{S} \\
(\mathrm{m} / \mathrm{m})\end{array}$ & $\begin{array}{c}\mathbf{U}^{*} \\
(\mathrm{~m} / \mathrm{s})\end{array}$ \\
\hline 1 & Rio das Velhas / D8 & $6 / 26 / 2001$ & $\mathrm{RO} / 4,7$ & 13,90 & 22,01 & 32,50 & 0,54 & 1,30 & 0,001 & 0,09 \\
2 & Rio das Velhas / DPRS & $6 / 26 / 2001$ & $\mathrm{RO} / 44,6$ & 24,80 & 48,54 & 49,50 & 0,53 & 1,90 & 0,001 & 0,11 \\
\hline 3 & Rio Paraibuna / Pontilhão & $10 / 7 / 2005$ & $\mathrm{AMl} / 16,0$ & 11,00 & 19,57 & 26,70 & 0,53 & 1,40 & 0,001 & 0,09 \\
\hline 4 & Rio Pomba / Sinimbu & $5 / 27 / 2006$ & $\mathrm{AMl} / 22,0$ & 29,00 & 44,30 & 49,00 & 0,40 & 2,20 & 0,001 & 0,10
\end{tabular}

Q: vazão; B: largura; U: velocidade média do escoamento; H: profundidade; S: declividade do curso d'água; u*: velocidade de cisalhamento.

Nesse método, se a razão de discrepância $\left(\mathrm{R}_{\mathrm{d}}\right)$ for igual a zero, a predição do valor do $D_{L}$ é idêntica ao coeficiente de dispersão medido. Se a razão de discrepância é maior que zero, a predição do coeficiente de dispersão é superestimada, e se a razão de discrepância é menor do que zero, é subestimada. Quanto maior o valor da razão de discrepância $\left(\mathrm{R}_{\mathrm{d}}\right)$, mais distante está o conjunto dos dados estimados $\left(E_{i}\right)$ dos valores reais observados $\left(\mathrm{O}_{\mathrm{i}}\right)$.

A REMQ é obtida pela Equação 7.

REMQ $=\sqrt{\frac{1}{N} \sum_{i=1}^{N}\left[E_{i}-O_{i}\right]^{2}}$ Equação 7

O terceiro método comparativo utilizado foi o índice de desempenho (c), proposto por Camargo e Sentelhas (1997). Nesse método, ao correlacionar os valores estimados $\left(\mathrm{E}_{\mathrm{i}}\right)$ com os medidos $\left(\mathrm{O}_{\mathrm{i}}\right)$, utilizando-se regressão linear, são considerados os seguintes indicadores estatísticos: "precisão" - coeficiente de correlação (r) e "exatidão" índice de Willmott (d).

A precisão é dada pelo coeficiente de correlação, em que os valores absolutos do coeficiente indicam o grau de dispersão dos dados obtidos em torno da função de ajustamento (Milone, 2004). Vale ressaltar que o coeficiente de correlação (r) varia entre -1 e 1. Uma correlação próxima de zero indica que as duas variáveis não estão relacionadas. Uma correlação positiva indica que as duas variáveis "movem-se na mesma direção" (diretamente proporcionais) e a relação é forte quanto mais o valor se aproxima de 1. Uma correlação negativa indica que as duas variáveis "movem-se em direções opostas" (inversamente proporcionais) e a relação é mais forte quanto mais próxima de -1 . Se as variáveis estão perfeitamente correlacionadas positivamente movem-se essencialmente em perfeita proporção na mesma direção, enquanto dois conjuntos que estão perfeitamente correlacionados negativamente movem-se em perfeita proporção em direções opostas (FONSECA; MARTINS; TOLEDO, 1985).

A exatidão está relacionada ao afastamento dos valores estimados em relação aos observados. Matematicamente, essa aproximação é dada por um índice designado concordância, representado pela letra "d" (WILLMOTT et al, 1985). Seus valores variam de 0, para nenhuma concordância, a 1, para a concordância perfeita. Valores de d acima de 0,75 são considerados satisfatórios. O índice é dado pela Equação 8:
$d=1-\left[\frac{\sum_{i=1}^{n}\left(E_{i}-O_{i}\right)^{2}}{\sum_{i=1}^{n}\left(\left|E_{i}-\bar{O}\right|+\left|O_{i}-\bar{O}\right|\right)^{2}}\right]$

Equação 8

Onde:

d: índice de concordância;

$\overline{\mathrm{O}}$ : média dos valores observados.

Isso posto, o índice c, representado pela Equação 9, é dado pelo produto do índice de precisão, correspondente ao coeficiente de correlação (r), com o índice de concordância (d) proposto por Willmott et al (1985), sendo os critérios de análise do desempenho apresentados na Tabela 4.

$c=r \cdot d$

Equação 9

\section{Resultados e discussão}

O desenvolvimento da equação de predição do $\mathrm{D}_{\mathrm{L}}$ a partir de resultados experimentais com traçador, a validação e a comparação do desempenho de diversas equações empíricas são apresentados na sequência.

\section{Obtenção da equação empírica}

Os parâmetros estimados por regressão linear múltipla foram: $\beta_{0}$ $=1,99 \times 10^{-4}, \beta_{1}=0,445, \beta_{2}=-1,458$ e $\beta_{3}=0,761$, resultando na seguinte relação matemática:

$\frac{\mathrm{D}_{\mathrm{L}}}{\mathrm{u}^{*} \mathrm{H}}=1,99.10^{-4}\left(\frac{\mathrm{B}}{\mathrm{H}}\right)^{0,445}\left(\frac{\mathrm{u}^{*}}{\mathrm{U}}\right)^{-1,458}(\mathrm{Re})^{0,761}$

Equação 10

Considerando-se o valor da viscosidade cinemática da água ( $v)$ para temperaturas próximas de $20^{\circ} \mathrm{C}$ igual a $1 \times 10^{-6} \mathrm{~m}^{2} \mathrm{~s}^{-1}$ e substituindo $\operatorname{Re}^{*}=\frac{\mathrm{u}^{*} . \mathrm{H}}{\mathrm{n}}$, a Equação 10 pode ser reescrita em função de $\mathrm{D}_{\mathrm{L}}$, conforme apresenta a Equação 11 .

$\mathrm{D}_{\mathrm{L}}=7,326 \cdot\left(\mathrm{u}^{*}\right)^{0,303} \cdot(\mathrm{H})^{1,316} \cdot(\mathrm{B})^{0,445} \cdot(\mathrm{U})^{1,458} \quad$ Equação 11

A Equação 11 permite quantificar a dispersão (ou espalhamento) de poluentes solúveis em rios de médio porte. Para verificar a qualidade 
da equação, ajustada à base de dados experimentais (rios Pomba e Paraibuna), apresentada na Tabela 1 , foi realizada uma avaliação do índice de determinação $\left(\mathrm{r}^{2}\right)$ e aplicado o teste estatístico $\mathrm{F}$ ao resultado da regressão. $\mathrm{O}$ índice de determinação encontrado $\left(r^{2}=0,85\right)$ demonstra que $85 \%$ da variação de $\mathrm{D}_{\mathrm{L}}$ é explicada pela equação de regressão, o que indica que a equação linear é adequada. Ao nível de significância $\alpha=5 \%$, foi rejeitada a hipótese de nulidade dos parâmetros, indicando que a regressão pode ser aceita com 95\% de confiança.

\section{Validação e comparação do desempenho da equação desenvolvida}

O processo de validação da equação desenvolvida (Equação 11) deu-se por meio da comparação com os resultados de $\mathrm{D}_{\mathrm{L}}$ obtidos por Bandeira (2004) e Ribeiro et al (2008). Concomitantemente, realizouse a comparação da Equação 11, ajustada no presente trabalho, com outras quatro equações empíricas propostas na literatura, na qual foi avaliado o desempenho relativo das diversas equações para estimar o $\mathrm{D}_{\mathrm{L}}$ com base nas quatro observações com traçador (Tabela 3). Desse

Tabela 4 - Critérios para análise do desempenho (c) de um modelo, segundo Camargo e Sentelhas (1997)

\begin{tabular}{lc} 
Valor de $c$ & Desempenho \\
$>0,85$ & Ótimo \\
0,76 a 0,85 & Muito Bom \\
0,66 a 0,75 & Bom \\
0,61 a 0,65 & Mediano \\
0,51 a 0,60 & Sofrível \\
0,41 a 0,50 & Mau \\
$\leq 0,40$ & Péssimo \\
\hline
\end{tabular}

modo, a comparação do desempenho entre as diversas equações empíricas e a equação desenvolvida neste estudo aconteceu nas mesmas condições hidráulicas do escoamento, permitindo, assim, avaliar os seus desempenhos em condições de campo semelhantes. Os resultados dos valores observados (Oi) e estimados (Ei) pelas diferentes equações são apresentados na Tabela 5 .

Na Tabela 6, estão apresentados os resultados das comparações entre os valores de $\mathrm{D}_{\mathrm{L}}$ observados $\left(\mathrm{O}_{\mathrm{i}}\right)$ e estimados $\left(\mathrm{E}_{\mathrm{i}}\right)$, utilizando-se o método comparativo da razão de discrepância $\left(\mathrm{R}_{\mathrm{d}}\right)$.

Os resultados apresentados na Tabela 6 evidenciam que a Equação 11, desenvolvida neste estudo, apresentou melhor desempenho quando comparada às demais $\left(\overline{R_{d}}=-0,16\right)$. Essa equação apresentou melhor aproximação do conjunto de valores estimados quando comparada ao conjunto dos valores observados. Verifica-se também nos resultados da Tabela 6 que a equação proposta por Seo e Cheong (1998) foi a que mais superestimou a predição dos valores de $D_{L}\left(\overline{R_{d}}=0,58\right)$ e a proposta por Devens, Barbosa Junior e Silva (2006) subestimou expressivamente os valores de $\mathrm{D}_{\mathrm{L}}$.

Os resultados do segundo método comparativo de desempenho utilizado, REMQ, estão apresentados na Tabela 7.

Verifica-se na Tabela 7 que a Equação 11, desenvolvida neste estudo, apresentou melhor desempenho $(\overline{\mathrm{REMQ}}=7,70)$ por esse método comparativo, quando comparada às demais.

Na Tabela 8, apresenta-se o resultado do índice de desempenho (CAMARGO; SENTELHAS, 1997) entre as diversas equações de predição do $\mathrm{D}_{\mathrm{L}}$.

Os resultados apresentados na Tabela 8 comprovam que a Equação 11 apresentou o melhor índice de desempenho ( $c=0,50)$ entre as equações de predição de $\mathrm{D}_{\mathrm{L}}$ avaliadas.

Tabela 5 - Valores do coeficiente de dispersão longitudinal $\left(D_{L}\right)$ observados (Oi) por Bandeira (2004) e Ribeiro et al. (2008) e estimados ( $\left.E_{i}\right)$ pelas diversas fórmulas empíricas

\begin{tabular}{|c|c|c|c|c|c|c|}
\hline \multirow[b]{2}{*}{ Obs. } & \multirow{2}{*}{$\begin{array}{c}\text { Valores } \\
\text { Observados (Oi) } \\
\text { Bandeira (2004) } \\
\text { e Ribeiro et al. (2008) }\end{array}$} & \multicolumn{5}{|c|}{ Valores Estimados (Ei) } \\
\hline & & $\begin{array}{c}\text { Seo\&Cheong } \\
(1998)\end{array}$ & $\begin{array}{l}\text { Deng et al. } \\
\text { (2001) }\end{array}$ & $\begin{array}{l}\text { Kashefipour\&Falconer } \\
\text { (2002) }\end{array}$ & $\begin{array}{l}\text { Devens et al. } \\
\qquad(2006)\end{array}$ & Eq.(15) \\
\hline 1 & 13,90 & 66,76 & 59,24 & 46,16 & 0,006 & 9,51 \\
\hline 2 & 24,80 & 88,42 & 78,21 & 52,55 & 0,005 & 19,15 \\
\hline 3 & 11,00 & 59,42 & 47,15 & 48,46 & 0,005 & 9,27 \\
\hline 4 & 29,00 & 63,48 & 49,96 & 35,96 & 0,004 & 15,47 \\
\hline
\end{tabular}

Tabela 6 - Resultados obtidos pelo método comparativo da razão de discrepância $\left(R_{d}\right)$ entre os valores de $D_{\perp}$ observados $\left(0_{i}\right)$ por Bandeira $(2004)$ e Ribeiro et al. (2008) e estimados $\left(\mathrm{E}_{\mathrm{i}}\right)$ pelas diversas fórmulas empíricas

\begin{tabular}{lccccc}
\hline & \multicolumn{4}{c}{$E_{i}$} \\
$O_{i}$ & Seo e Cheong (1998) & $\begin{array}{c}\text { Deng, Singh e Bengts- } \\
\text { son (2001) }\end{array}$ & $\begin{array}{c}\text { Kashefipour e Falconer } \\
(2002)\end{array}$ & $\begin{array}{c}\text { Devens, Barbosa Junior } \\
\text { e Silva (2006) }\end{array}$ & $\begin{array}{c}\text { Equação 15 } \\
1\end{array}$ \\
\hline 2 & 0,68 & 0,63 & 0,52 & $-3,36$ & $-0,17$ \\
3 & 0,55 & 0,50 & 0,33 & $-3,74$ & $-0,11$ \\
4 & 0,73 & 0,63 & 0,64 & $-3,37$ & $-0,07$ \\
$R_{d}$ média & 0,34 & 0,24 & 0,09 & $-3,81$ & $-0,27$ \\
\hline
\end{tabular}


Segundo o critério proposto por Camargo e Sentelhas (1997), o modelo desenvolvido neste trabalho (Equação 11) enquadra-se na classe de mau desempenho $(c=0,50)$ e índice de concordância não satisfatório $(d=0,58)$. Porém, quando comparado com o desempenho dos outros modelos ( $c \leq 0,18$ e $\mathrm{d} \leq 0,40$ ) - conforme apresenta a Tabela 8, utilizando-se os mesmos critérios de classificação, ao partir do desempenho apresentado e do índice de concordância entre os valores estimados e observados - verifica-se que ainda é bastante superior aos demais modelos. Vale destacar que em todos os testes comparativos, mesmo no método proposto por Camargo e Sentelhas (1997), no qual $r=0,86$, o modelo desenvolvido (Equação 11) apresentou boa correlação entre os valores de $D_{L}$ estimados e os observados experimentalmente nos rios Paraibuna, Pomba e rio das Velhas, sugerindo que esse modelo estima satisfatoriamente o valor de dispersão longitudinal para a faixa de vazões consideradas neste trabalho.

As diferenças expressivas nos resultados de desempenho apresentados pelas diversas fórmulas empíricas podem ser explicadas pelo fato de que essas equações foram deduzidas para condições específicas, conforme apresentado na Tabela 2, podendo gerar distorções quando aplicadas a condições hidráulicas de escoamento diferentes daquelas consideradas em sua concepção. Nesse sentido, a equação proposta neste estudo, desenvolvida para faixa de velocidades e profundidade média de 0,50 a 0,92 m/s e 1,17 a 2,42 m, respectivamente, apresentou melhor desempenho quando comparada com a base de dados de experimentais dos estudos de Bandeira (2004) e Ribeiro et al (2008), apresentados na Tabela 3, em que os valores de velocidade média e profundidade variam de 0,40 a $0,54 \mathrm{~m} / \mathrm{s}$ e 1,30 a 2,20 $\mathrm{m}$, respectivamente.

Com exceção da equação proposta por Devens, Barbosa Junior e Silva (2006) - idealizada para cursos d'água de pequeno porte, com faixa de velocidade média de escoamento e profundidade de 0,08 a
$0,34 \mathrm{~m} / \mathrm{s}$ e 0,02 a $0,10 \mathrm{~m}$, respectivamente, a qual subestimou significativamente os valores de predição de $D_{L}$ - as demais equações apresentaram faixa de velocidade e profundidade média compatíveis com os valores utilizados na concepção da Equação 11, proposta neste trabalho.

\section{Conclusões}

A equação de predição do $\mathrm{D}_{\mathrm{L}}$, desenvolvida no presente trabalho, calibrada para a faixa de vazões de 16,20 a $98 \mathrm{~m}^{3} \mathrm{~s}^{-1}$ e validada para vazões entre 19,57 a $48,54 \mathrm{~m}^{3} \mathrm{~s}^{-1}$, apresentou ajuste estatístico adequado e bom desempenho quando testada em outra base de dados, diferente daquela para a qual foi desenvolvida.

Verifica-se que, embora o desempenho da equação não tenha sido plenamente satisfatório pelo critério de Camargo e Sentelhas (1997), todos os outros testes comparativos apontaram uma boa correlação entre os dados estimados e os observados experimentalmente, sugerindo um bom desempenho da equação desenvolvida para a estimativa do $\mathrm{D}_{\mathrm{L}}$.

Quando comparada às outras quatro equações propostas na literatura para a predição do $D_{L}$, a equação proposta no trabalho apresentou o melhor desempenho geral.

Os resultados obtidos contribuem expressivamente para subsidiar estudos de gerenciamento e modelagem da qualidade da água em rios de médio porte, facilitando a quantificação do coeficiente de dispersão para a simulação do transporte de contaminantes solúveis.

\section{Agradecimentos}

Ao CNPq e à FAPEMIG, pelo aporte financeiro, e à CAPES, pela concessão da bolsa de Doutorado, que possibilitou a realização dos trabalhos

Tabela 7 - Resultados obtidos pelo método comparativo da raiz do erro médio quadrático (REMQ) entre os valores do coeficiente de dispersão longitudinal $\left(D_{L}\right)$ observados $\left(0_{i}\right)$ por Bandeira (2004) e Ribeiro et al. (2008) e estimados $\left(E_{i}\right)$ pelas diversas fórmulas empíricas

\begin{tabular}{|c|c|c|c|c|c|}
\hline \multirow[b]{2}{*}{$\mathrm{O}_{\mathrm{i}}$} & \multicolumn{5}{|c|}{$E_{i}$} \\
\hline & Seo e Cheong (1998) & $\begin{array}{c}\text { Deng, Singh e } \\
\text { Bengtsson (2001) }\end{array}$ & $\begin{array}{l}\text { Kashefipour e Falconer } \\
\text { (2002) }\end{array}$ & $\begin{array}{c}\text { Devens, Barbosa Junior } \\
\text { e Silva (2006) }\end{array}$ & Equação 15 \\
\hline 1 & $2.793,93$ & $2.055,94$ & $1.040,62$ & 193,04 & 19,31 \\
\hline 2 & $4.046,95$ & $2.852,39$ & 770,13 & 614,81 & 31,93 \\
\hline 3 & $2.344,93$ & $1.306,66$ & $1.403,24$ & 120,90 & 2,98 \\
\hline 4 & $1.189,14$ & 439,21 & 48,43 & 840,74 & 183,10 \\
\hline REMQ & 50,93 & 40,79 & 28,56 & 21,03 & 7,70 \\
\hline
\end{tabular}

Tabela 8 - Resultados do coeficiente de correlação (r), índice de Willmott (d) e índice de desempenho (c) para as diversas equações de predição de $D_{L}$

\begin{tabular}{lccc} 
Equação & r & d & 0,31 \\
Seo e Cheong (1998) & 0,43 & 0,13 & 0,36 \\
Deng, Singh e Bengtsson (2001) & 0,32 & 0,11 & 0,40 \\
Kashefipour e Falconer (2002) & $-0,45$ & 0,24 \\
Devens, Barbosa Junior e Silva (2006) & $-0,57$ & $-0,18$ & 0,58 \\
Equação 15 & 0,86 & 0,50 \\
\hline
\end{tabular}




\section{Referências}

BANDEIRA, J.V. Desenvolvimento de técnicas nucleares e correlatas para estudos em hidrologia urbana - aplicações na Bacia Hidrográfica da Pampulha e no Rio das Velhas, MG. Tese (Doutorado em Saneamento, Meio Ambiente e Recursos Hídricos), Universidade Federal de Minas Gerais, Belo Horizonte, MG, 2004.

BOWIE, G.L. et al. Rates, constants, and kinetics formulations in surface water quality modeling, 2. ed., Environmental Research Laboratory, Office of Research and Development, U.S. Environmental Protection Agency, 1985.

CAMARGO, A.P. SENTELHAS, P.C. Avaliação do desempenho de diferentes métodos de estimativa da evapotranspiração potencial no Estado de São Paulo, Brasil. Revista Brasileira de Agrometeorologia, v. 5, n. 1, p. 89-97, 1997.

DENG, Z.Q.; SINGH, V.P.; BENGTSSON, L. Longitudinal dispersion coefficient in straight rivers. Journal of Hydraulic Engineering, v. 127, n. 11, p. 919-927, 2001.

DEVENS, J.A.; BARBOSA JUNIOR, A.R.; SILVA, G.Q. Modelo de quantificação do coeficiente de dispersão longitudinal de pequenos cursos de água naturais. Revista Engenharia Sanitária e Ambiental, v. 11, n. 3, p. 269-276, 2006

FONSECA, J.S.; MARTINS, G.A.; TOLEDO, G.L. Estatística aplicada. 2. ed., São Paulo: Atlas, 1995.

KASHEFIPOUR, S.M.; FALCONER, R.A. Longitudinal dispersion coefficients in natural channels. Water Research, v. 36, n. 6, p. 15961608, 2002

MILONE, G. Estatística geral e aplicada. São Paulo: Pioneira Thomson Learning, 2004
PEREIRA, C.B. Influência do método de estimativa do coeficiente de dispersão longitudinal na simulação da qualidade da água em rios. Dissertação (Mestrado em Engenharia Ambiental), Universidade Federa do Espírito Santo Vitória, ES, 1999.

RIBEIRO, C.B.M. et al. Determinação "in situ" do coeficiente de dispersão longitudinal utilizando técnicas de traçadores. In: Simpósio de Recursos Hídricos da Bacia do Rio Paraíba do Sul, 1, Anais..., Resende, 2008.

Comparação de metodologias para determinação do coeficiente de dispersão longitudinal em rios: estudo desenvolvido no Rio Pomba (MG). In: Simpósio Brasileiro de Recursos Hídricos, 17, Anais..., São Paulo: ABRH, 2007.

RIECKERMANN, J. et al. Dispersion coefficients of sewers tracer experiments. In: International Conference on Urban Drainage Modeling, 6. Anais..., Dresden, Germany, p. 417-426, 2004.

SEO, W.; CHEONG, T.S. Predicting longitudinal dispersion coefficient in natural streams. Journal of Hydraulic Engineering, v. 124, n. 1, p. 25-32, 1998

TAYFUR, G.; SINGH, V.P. Predicting longitudinal dispersion coefficient in natural streams by artificial neural network. Journal of Hydraulic Enginnering, v. 131, n. 11, p. 991-1000, 2005

WHITE, W.R.; MILLI, H.; CRABBE, A.D. Sediment transport: an appraisal methods. Vol. 2: performance of theoretical methods when applied to flume and field data. Hydraulics Research Station Report No IT 119 Wallingford, U.K., 1973

WILLMOTT, C.J. et al. Statistics for the evaluation and comparison of models. Journal of Geophysical Research, v. 90, n. C5, p. 8995-9005, 1985 